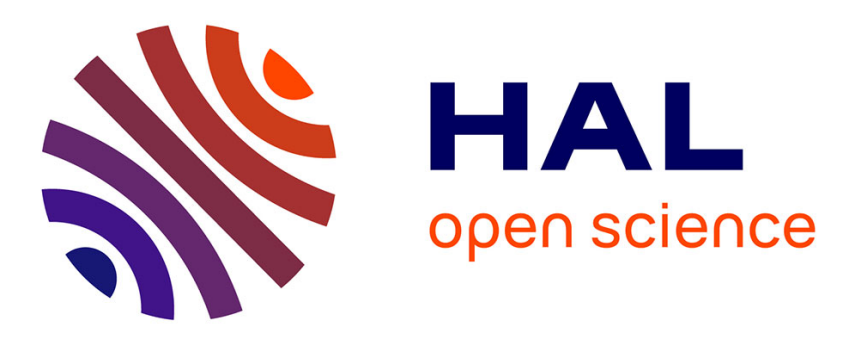

\title{
Numerical and experimental study of multi-contact on an elastic half-space
}

Julien Cesbron, Honoré P. Yin, Fabienne Anfosso-Lédée, Denis Duhamel, Donatien Le Houedec, Zi Qiang Feng

\section{- To cite this version:}

Julien Cesbron, Honoré P. Yin, Fabienne Anfosso-Lédée, Denis Duhamel, Donatien Le Houedec, et al.. Numerical and experimental study of multi-contact on an elastic half-space. International Journal of Mechanical Sciences, 2009, 51 (1), pp.33-40. 10.1016/j.ijmecsci.2008.11.003 . hal-01007123

\section{HAL Id: hal-01007123 \\ https://hal.science/hal-01007123}

Submitted on 13 Dec 2017

HAL is a multi-disciplinary open access archive for the deposit and dissemination of scientific research documents, whether they are published or not. The documents may come from teaching and research institutions in France or abroad, or from public or private research centers.
L'archive ouverte pluridisciplinaire HAL, est destinée au dépôt et à la diffusion de documents scientifiques de niveau recherche, publiés ou non, émanant des établissements d'enseignement et de recherche français ou étrangers, des laboratoires publics ou privés. 


\title{
Numerical and experimental study of multi-contact on an elastic half-space
}

\author{
J. Cesbron ${ }^{\text {a,* }}$, H.P. Yin ${ }^{\text {b }}$, F. Anfosso-Lédée ${ }^{\text {a }}$, D. Duhamel ${ }^{\text {b }}$, D. Le Houédec ${ }^{c}$, Z.Q. Feng ${ }^{d}$ \\ ${ }^{a}$ LCPC, BP 4129, 44341 Bouguenais Cedex, France \\ ${ }^{\mathrm{b}}$ Université Paris-Est, UR Navier, ENPC, 6 et 8 Avenue Blaise Pascal, Cité Descartes, Champs sur Marne, 77455 Marne la Vallée Cedex 2, France \\ c ECN, GeM, 1 rue de la Noë, BP 92101, 44321 Nantes Cedex 3, France \\ ' LMEE, Université d'Evry, 40 rue du Pelvoux, 91020 Evry, France
}

This paper deals with the numerical and experimental study of multiple contacts at the surface of an elastic half-space. A two-scale iterative method is proposed for solving the problem. First a procedure that takes into account interaction gives the contact forces at the tips of the asperities from which the pressure distribution at the contact interface is then calculated using an iterative scheme. Numerical results in the case of two and seven spherical indentors show that the method is as accurate as classical methods and very time efficient even for close proximity contacts. Additionally contact forces and pressures between a rubber block and several spherical indenters were measured. The differences between experiments and theoretical predictions were below $10 \%$. This means that the proposed method can be a reliable tool to model contact problems for which such an accuracy is enough.
Keywords:

Contact mechanics

Numerical methods

Experimental methods

\section{Introduction}

The contact between an elastic half-space and multiple rigid indentors is of great interest in many engineering applications. This was for instance used in $[1,2]$ to model the dynamical contact stresses between the tyre tread and the road for rolling noise prediction.

The normal contact between a rigid surface and an elastic half-space was first investigated by Boussinesq [3] using the potential theory. Numerous analytical solutions were then derived for a single indentor of axisymmetric profile by Sneddon [4]. For a large number of indentors, Greenwood and Williamson [5] developed a statistical spherical asperity-based model. A deterministic numerical approach was also developed by using some known half-space solutions to construct influence coefficients. It can be solved using the matrix inversion method of Johnson [6] or the optimization method of Kalker [7]. It was widely used and improved in [8-11]. However, these methods can become time-consuming when the number of indentors increases.

This paper presents a new technique for solving the problem of multi-contact on an elastic half-space. It solves the problem in two steps and is named the two-scale iterative method. The first step consists in calculating the contact forces at the tips of the indentors. The procedure is similar to the one proposed in $[12,13]$ but is valid here for three-dimensional problems and indentors of

\footnotetext{
* Corresponding author. Tel.: +332408456 62; fax: +33240845992.

E-mail address: julien.cesbron@lcpc.fr (J. Cesbron).
}

random shape. The second step of the method gives the pressure distribution at the contact interface using an iterative scheme that starts from the contact forces obtained at the first step. After having described the method, its efficiency in the case of close proximity contacts will be demonstrated from dimensionless numerical results involving spherical punches. Then experimental contact data measured between spherical indentors and a rubber block will be compared to predicted values obtained with the method. The measurements are carried out using a digital pressure sensing device which was successfully used in the literature to compare numerical models with experimental contact data in [14-16]. Finally, the correlations between the measurements and the calculations will be discussed before concluding remarks.

\section{Two-scale iterative method for multi-contact on a half-space}

The considered problem is illustrated in Fig. 1 and is governed by the following relations of unilateral contact:

$$
\begin{aligned}
& \forall(x, y) \in \Sigma, u(x, y)=\iint_{\Sigma_{c}} p(\xi, \eta) T(x, y ; \xi, \eta) \mathrm{d} \xi \mathrm{d} \eta \\
& \forall(x, y) \in \Sigma_{c}, u(x, y)=\delta-z(x, y) \text { and } p(x, y)>0
\end{aligned}
$$

where $z$ describes the height of the rigid surface, $\delta$ is the global penetration between the contacting bodies, $\Sigma$ is the surface of the half-space, $\Sigma_{c}$ is the contact area, $u$ is the displacement at the surface of the half-space and $p$ is the normal pressure at the contact interface. In the integral of Eq. (1), the influence function $T$ 


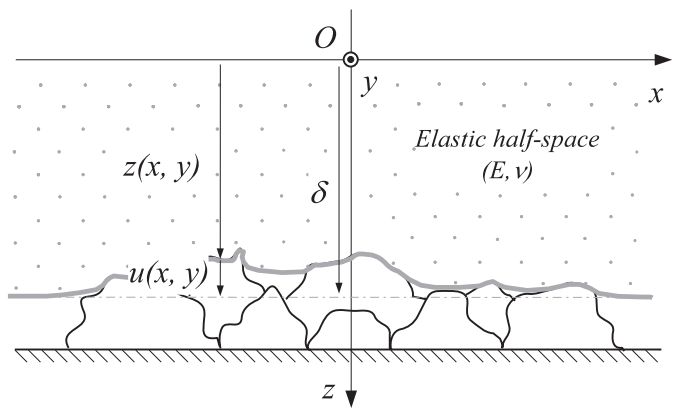

Fig. 1. Elastic half-space in contact with several rigid punches.

is defined by Boussinesq's theory [3]:

$\forall\left(x, y ; x^{\prime}, y^{\prime}\right) \in \Sigma^{2}, \quad T\left(x, y ; x^{\prime}, y^{\prime}\right)=\left[\pi E^{*} \sqrt{\left(x-x^{\prime}\right)^{2}+\left(y-y^{\prime}\right)^{2}}\right]^{-1}$

with $E^{*}=E /\left(1-v^{2}\right)$ where $E$ is the Young modulus of the halfspace and $v$ is its Poisson's coefficient. Eq. (2) contains the two conditions of non-penetration and of compressive contact between the bodies. If the global penetration $\delta$ is given, the unknowns of the contact problem are the contact area $\Sigma_{c}$ and the pressure distribution $p$.

The resolution of Eqs. (1) and (2) can be very time consuming when the number of asperities becomes large. So we propose to solve this problem in two steps, as illustrated in Fig. 2. The first step, denoted the macro-scale calculation, consists in an approximate problem in which only the global forces acting at the tips of the asperities are computed (Fig. 2(a)). Then, this solution is used in a second step, denoted the micro-scale calculation, to compute the pressure distribution in the whole contact area (Figs. 2(b) and (c)).

\subsection{The macro-scale calculation step}

Considering that the indenting surface is composed of $N$ asperities with tips of coordinates $\left(x_{k}, y_{k}, z_{k}\right)$, then the contact forces $P_{k}$ at the summit of the punches can be written as

$\forall k \in[1, N], \quad P_{k}=f_{k}\left[\delta_{k} H\left(\delta_{k}\right)\right]$

where $H$ is Heaviside's function defined by $H\left(\delta_{k}\right)=0$ if $\delta_{k}<0$ and $H\left(\delta_{k}\right)=1$ if $\delta_{k} \geqslant 0 . f_{k}$ is the load-penetration function obtained for the $k$ th punch alone. It is assumed that the multiple contacts have few influence on this function. The local penetration $\delta_{k}$ corresponds to the displacement of the half-space at the summit of the $k$ th punch. If only the asperity $k$ acts at the surface of the halfspace, then $\delta_{k}$ is simply equal to $\delta-z_{k}$. In the multi-contact case, each force $P_{l}$ that acts at the tips of another punch $l$ will induce a displacement at punch $k$ given by $T_{k l} P_{l}$ with $T_{k l}=T\left(x_{k}, y_{k} ; x_{l}, y_{l}\right)$. According to the superposition principle in linear elasticity theory, the local penetration $\delta_{k}$ can then be written as

$\delta_{k}=\delta-z_{k}-u_{k}=\delta-z_{k}-\sum_{\substack{l=1 \\ l \neq k}}^{N} T_{k l} P_{l}$

Introducing relation (5) in Eq. (4) lead to a non-linear system of $N$ equations with $N$ unknowns $P_{k}$. It is solved using the Newton-Raphson iterative method and gives the contact forces $P_{k}$ at the tips of the asperities.

\subsection{The micro-scale calculation step}

Then the surface of the elastic half-space is divided in $n$ identical square elements with coordinates $\left(x_{i}, y_{i}, z_{i}\right)$ and size a

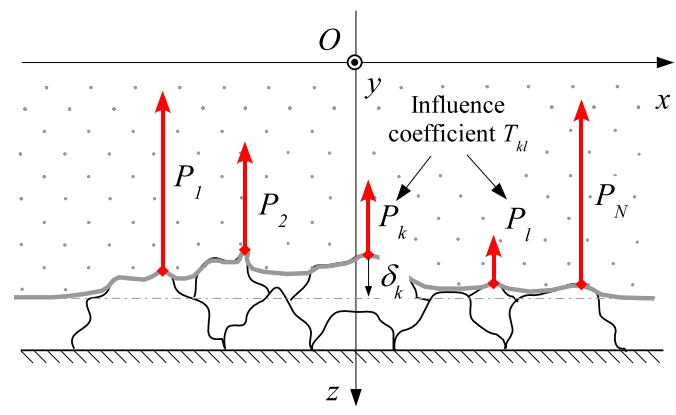

b

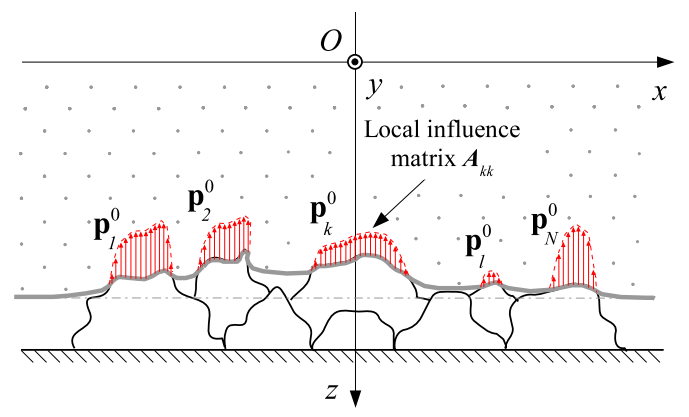

C

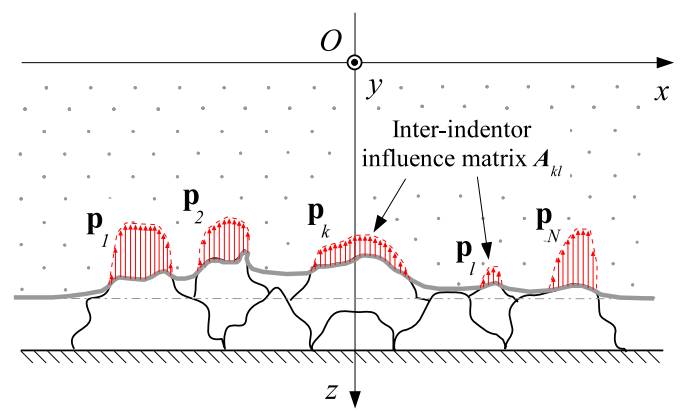

Fig. 2. Schematic view of the two-scale iterative method. (a) Macro-scale calculation step: contact force $P_{k}$ on tips; (b) micro-scale calculation step: first pressure approximation $\mathbf{p}^{0}$; (c) micro-scale calculation step: final pressure distribution $\mathbf{p}$.

$h \times h$. A global influence matrix, noted $\mathbf{A}$, is defined. Its coefficients are calculated using the analytical results of Love [17] assuming that a uniform pressure is acting on each square element. Defining a global pressure vector $\mathbf{p}=\left\{p_{i}\right\}_{i \in[1, n]}^{\mathrm{T}}$ and a displacement vector $\mathbf{b}=\left\{\delta-z_{i}\right\}_{i \in[1, n]}^{\mathrm{T}}$ then the contact problem of Eqs. (1) and (2) is written as a vectorial equation $\mathbf{A p}=\mathbf{b}$. Matrix $\mathbf{A}$ is organized by blocks as follows:

$\left(\begin{array}{ccccc}\mathbf{A}_{11} & \cdots & \mathbf{A}_{1 k} & \cdots & \mathbf{A}_{1 N} \\ \vdots & \ddots & \vdots & \ddots & \vdots \\ \mathbf{A}_{k 1} & \cdots & \mathbf{A}_{k k} & \cdots & \mathbf{A}_{k N} \\ \vdots & \ddots & \vdots & \ddots & \vdots \\ \mathbf{A}_{N 1} & \cdots & \mathbf{A}_{N k} & \cdots & \mathbf{A}_{N N}\end{array}\right)\left\{\begin{array}{c}\mathbf{p}_{1} \\ \vdots \\ \mathbf{p}_{k} \\ \vdots \\ \mathbf{p}_{N}\end{array}\right\}=\left\{\begin{array}{c}\mathbf{b}_{1} \\ \vdots \\ \mathbf{b}_{k} \\ \vdots \\ \mathbf{b}_{N}\end{array}\right\}$

where $N$ is the number of indentors at the macro-scale, $\mathbf{A}_{k k}$ is the local influence matrix, $\mathbf{p}_{k}$ is the local pressure vector and $\mathbf{b}_{k}$ is the local displacement vector for the $k$ th punch. The extra-diagonal block $\mathbf{A}_{k l}$ is the part of $\mathbf{A}$ relative to the interaction of punch $l$ on punch $k$.

From the macro-scale forces $P_{k}$, an initial approximation of the contact pressure $\mathbf{p}^{0}=\left\{\mathbf{p}_{1}^{0}\left(P_{1}\right) \cdots \mathbf{p}_{k}^{0}\left(P_{k}\right) \cdots \mathbf{p}_{N}^{0}\left(P_{N}\right)\right\}^{\mathrm{T}}$ is calculated, 
with $\mathbf{p}_{k}^{0}$ such as

$$
\left(\begin{array}{cccc}
\mathrm{A}_{k_{11}} & \cdots & \mathrm{A}_{k_{1 n_{k}}} & -1 \\
\vdots & \ddots & \vdots & \vdots \\
\mathrm{A}_{k_{n_{k}} 1} & \cdots & \mathrm{A}_{k_{n_{k} n_{k}}} & -1 \\
-1 & \cdots & -1 & 0
\end{array}\right)\left\{\begin{array}{c}
p_{k_{1}}^{0} \\
\vdots \\
p_{k_{n_{k}}}^{0} \\
\delta-u_{k}
\end{array}\right\}=\left\{\begin{array}{c}
-z_{k_{1}} \\
\vdots \\
-z_{k_{n_{k}}} \\
-P_{k} / h^{2}
\end{array}\right\}
$$

where $A_{k_{i j}}$ are the elements of matrix $\mathbf{A}_{k k}$ and $n_{k}$ is the number of points on the asperity $k$. The initial pressure $\mathbf{p}_{k}^{0}$ on punch $k$ is obtained from Eq. (7) using the classical matrix inversion method of Johnson [6].

Then the contact problem is solved using a non-linear block Gauss-Seidel like algorithm. The iterative process starts from $\mathbf{p}^{0}$. At step $m+1$, the pressure distribution on each punch $k$, noted $\mathbf{p}_{k}^{m+1}$, is calculated by inverting the following local linear problem:

$\mathbf{A}_{k k} \mathbf{p}_{k}^{m+1}=\mathbf{b}_{k}^{m}-\sum_{l=1}^{k-1} \mathbf{A}_{k l} \mathbf{p}_{l}^{m+1}-\sum_{l=k+1}^{N} \mathbf{A}_{k l} \mathbf{p}_{l}^{m}$

After this the negative pressures obtained in $\mathbf{p}_{k}^{m+1}$ are replaced by zeros and the procedure is repeated on the other punches. The procedure is stopped when no negative pressure has been detected during the step and when the following convergence criteria is respected:

$\frac{\left\|\mathbf{p}^{m+1}-\mathbf{p}^{m}\right\|}{\left\|\mathbf{p}^{m}\right\|} \leqslant \varepsilon \quad$ with $\|\mathbf{X}\|=\sum_{i=1}^{n}\left|x_{i}\right|^{2}$

The convergence parameter $\varepsilon$ fixes the precision of the method.

Finally, the two-scale iterative method gives the solution to the global contact problem given by Eqs. (1) and (2). The proposed approach enables to reduce the number of iterations because the macro-scale step leads to a good initial pressure distribution $\mathbf{p}^{0}$ and because the resolution is simplified by applying the iterative formula of Eq. (8) instead of inverting the global influence matrix as in usual methods.

\section{Numerical results for multiple spherical indentors}

The goal of this numerical study is to show the efficiency of the proposed method even in close proximity contacts and to compare its results with those of classical numerical methods.

The two-scale iterative method was implemented for two configurations involving identical spherical indentors at the same height. The first one was composed of two spherical caps of radius
$R$ which summits are separate by the distance $d$. The second configuration involved seven spherical asperities of radius $R$, with six asperities at the distance $d$ from the last one and hexagonally distributed around it. For the contact forces calculation step, the load-penetration relation $f_{k}$ on each punch was given by Hertz's theory [18]:

$P_{k}=f_{k}\left(\delta_{k}\right)=4 / 3 E^{*} \sqrt{R} \delta_{k}^{3 / 2}$

with $E=10 \mathrm{MPa}$ and $v=0.5$ which corresponds to the elastic behavior of rubber-like materials. For the second step of the method, the surface of the half-space was meshed using square identical elements of length $h=0.0125 R$ for the two indentors case and $h=0.025 R$ for the seven indentors case.

Calculations were performed for several values of the parameter $d / 2 R$ at a fixed value of $\delta$. Examples of pressure distributions obtained for the two indentors case are given in Fig. 3 for $d / 2 R=0.1,0.25$ and 1.0 and $\delta=0.05 R$. Dimensionless pressure values $p / p_{m}$ are given, where $p_{m}$ is the mean pressure at the contact interface. The circles in dotted lines have a radius $R$ and indicate the position of the punches. These results clearly show the influence of the distance $d$ on the interaction between the two punches. When the indentors are very close, the local contact areas are no longer circular and the local pressure distributions become strongly asymmetrical. The same effects are observed in Fig. 4 for seven indentors at $d / 2 R=0.2$ and 1.0 and $\delta=0.1 R$. Additionally Fig. 5 gives the variation with $d / 2 R$ of the pressure distribution along the central radial line of the upper asperity for both configurations. This clearly shows that when the indentors are very far away $(d / 2 R=5)$ the local pressure
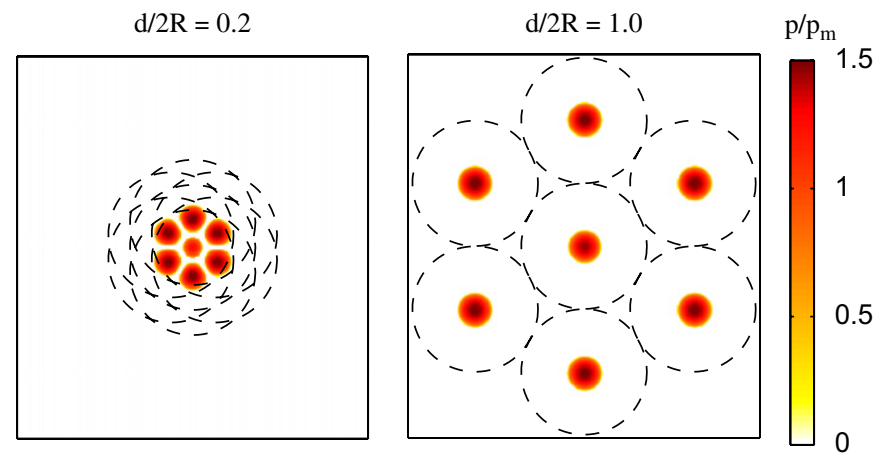

Fig. 4. Pressure distributions in the case of seven spherical indentors.
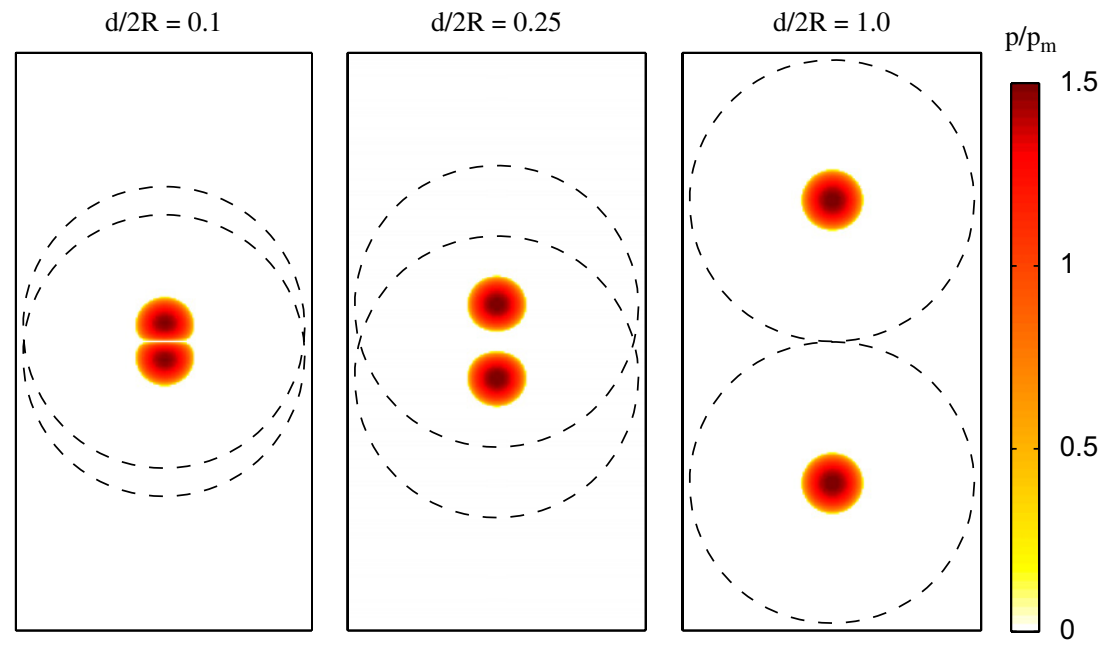

Fig. 3. Pressure distributions in the case of two spherical indentors. 

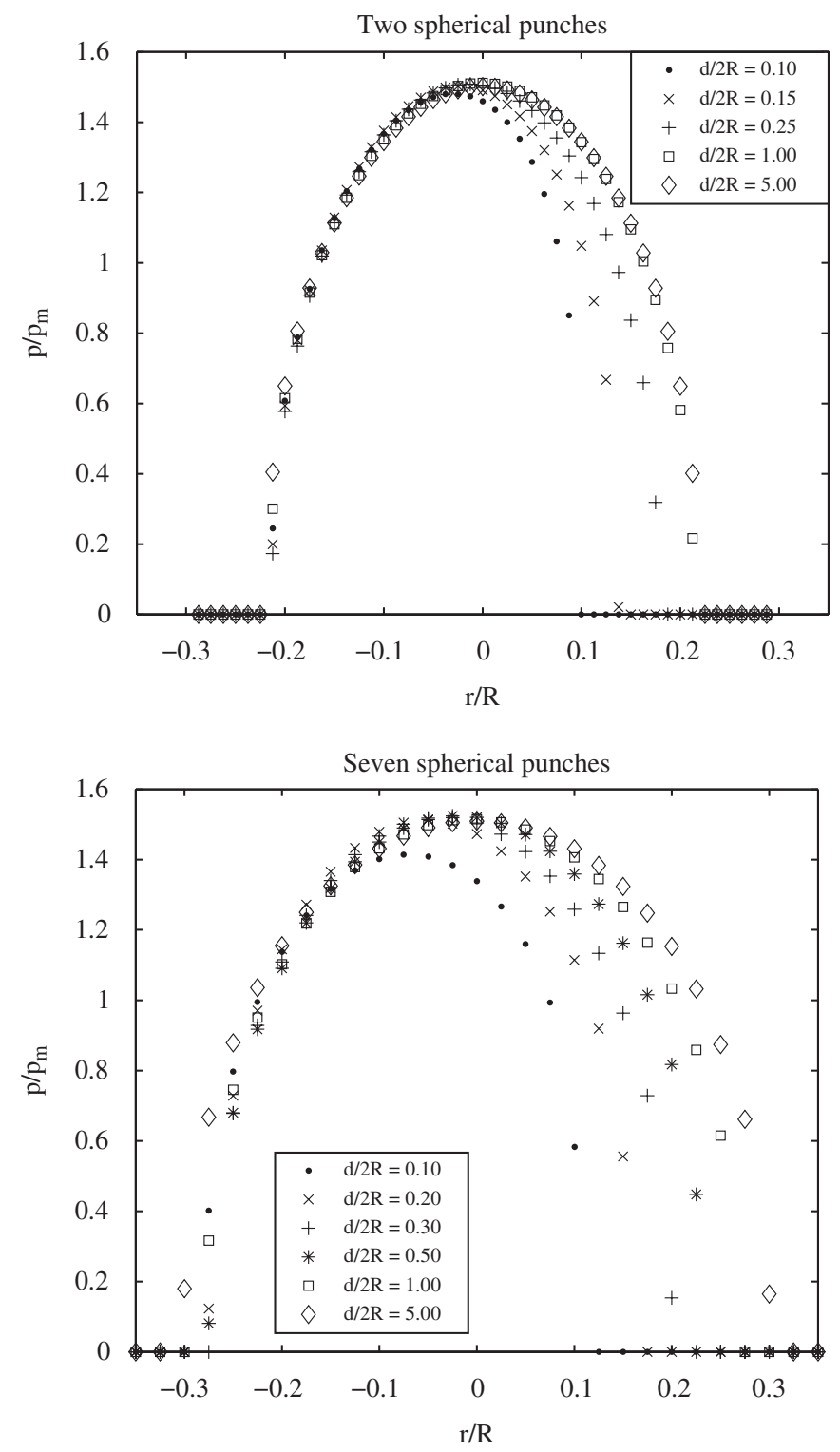

Fig. 5. Radial pressure on the central vertical axe for the top asperity.

distribution is symmetrical and very similar to the one obtained for a single spherical asperity. When the spheres are closer, the distribution becomes asymmetrical and is no longer centered around the tip of the punch.

The results presented above show the efficiency of the twoscale iterative method for dealing with multi-contact problems even for close proximity configurations. The accuracy of the method was estimated by comparing the initial and final pressure distributions, respectively $\mathbf{p}^{0}$ and $\mathbf{p}$, with the reference pressure distribution $\mathbf{p}_{r}$ obtained using the classical matrix inversion method of Johnson [6]. The differences in percents were estimated using the indicators $100\left\|\mathbf{p}^{0}-\mathbf{p}_{r}\right\| /\left\|\mathbf{p}_{r}\right\|$ and $100\left\|\mathbf{p}-\mathbf{p}_{r}\right\| /\left\|\mathbf{p}_{r}\right\|$. The variation of these indicators with $d / 2 R$ is represented in Fig. 6. In both configurations, the difference between the initial pressure $\mathbf{p}^{0}$ and the reference pressure $\mathbf{p}_{r}$ increases when the indentors become closer, but the final pressure $\mathbf{p}$ is always the same as the reference one. Then the two-scale iterative method leads to the same result as the classical method even for close proximity contact areas. Moreover, the first approximation $\mathbf{p}^{0}$ can give accurate results when the punches are sparse enough. Finally, the proposed method was about two and ten times faster than the
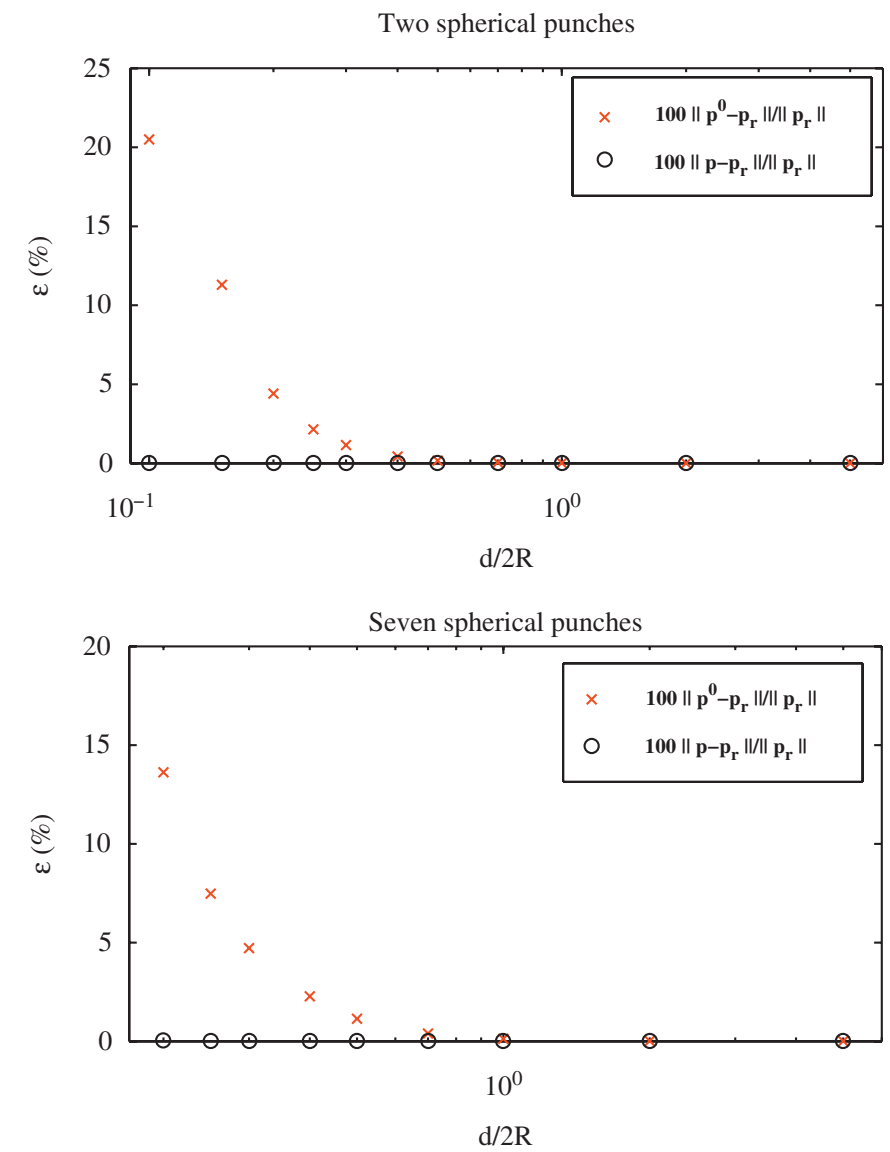

Fig. 6. Comparison of the two-scale iterative method ( $\mathbf{p}^{0}$ and $\mathbf{p}$ ) with the classical matrix inversion method $\mathbf{p}_{r}$.

classical method, respectively, for two and seven asperities and the iterative procedure of Eq. (8) converged in less than 10 iterations.

\section{Experimental comparisons}

The contact between a rubber block and surfaces composed of spherical indentors was experimentally studied for comparison with the proposed method. The purpose was to measure the contact forces and normal stresses at the contact interface and to compare them with the results of the model.

\subsection{Materials and method}

The bodies were brought into contact using a compression test machine as shown in Fig. 7. The total applied load was measured using a force transducer. The loading speed of the base plate was $V=1 \mathrm{~mm} \cdot \mathrm{min}^{-1}$ at which the viscoelasticity of the rubber was neglected. Plain bearings were introduced between the indenting surface and the punch of the press in order to spread out the contact forces properly over the whole contact area during the tests.

The normal stresses at the contact interface were measured in real time using a pressure sensor linked to a computer. This sensor is a resistive polymer divided in 1936 cells of $1.9 \mathrm{~mm}$ by $1.9 \mathrm{~mm}$ on which the electrical impedance varies with pressure and is converted into digital values between 0 and 255. Following the recommendations of the manufacturer [19], these raw data were 
calibrated from total load measured with the force transducer. It was assumed that the relation between the pressure and the digital output is linear and that zero output corresponds to null pressure. Crinkle artefacts also appeared during loading due to creasing of the sensor around the perimeter of the contact areas. These were treated by removing small raw values using a threshold which was fixed to 20. Finally, the overall system accuracy was $\pm 10 \%$ of the full pressure scale which varies between 0 and $3.5 \mathrm{MPa}$.

The dimensions of the rubber block $(140 \times 140 \times 20 \mathrm{~mm})$ were chosen large enough in comparison with those of the indenting

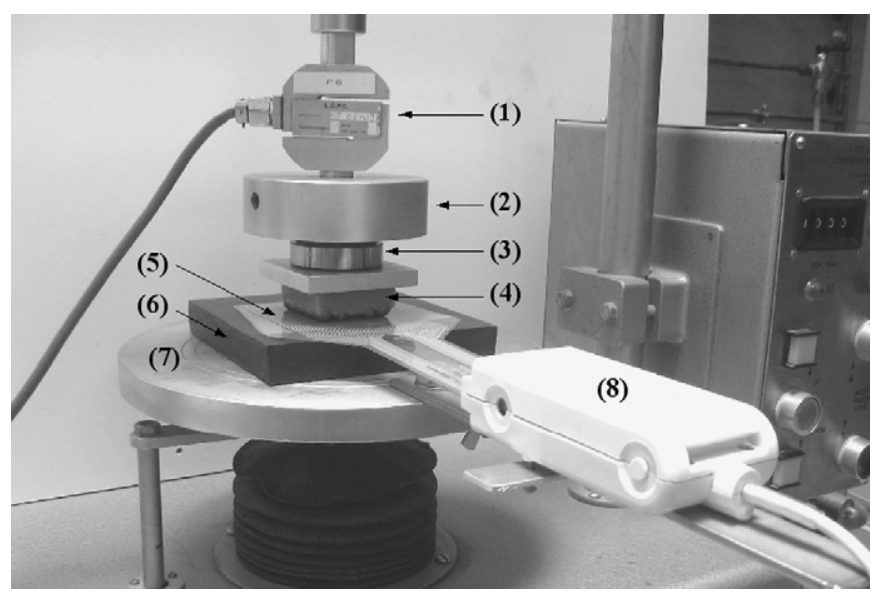

Fig. 7. Experimental setup: (1) force transducer, (2) punch of the press, (3) plain bearings, (4) indenting surface, (5) pressure sensor, (6) rubber block, (7) moving base plate of the press and (8) sensor handle linked to a PC. surface in order to fulfill the half-space hypothesis needed in the model. A Young's modulus $E=6 \mathrm{MPa}$ was identified using Hertz's theory [18] from a contact test between a single spherical indentor and the rubber block including the sensor at the interface. A Poisson's ratio $v=0.5$ was assumed. Three indenting surfaces named $S_{1}, S_{2}$ and $S_{3}$ were used for contact tests with the rubber block, as shown in Fig. 8. Each surface was composed of spherical asperities at the same height. The surface $S_{1}$ had seven identical indentors of radius $6 \mathrm{~mm}$ and hexagonally distributed. The surface $S_{2}$ was periodic and composed of 25 asperities of equal radius $(5 \mathrm{~mm})$. The surface $S_{3}$ had 24 indentors with random positions and three possible radii $(4,5$ or $6 \mathrm{~mm})$. The surfaces were steel-made and thus assumed perfectly rigid in comparison with the rubber block. For each surface, the asperities were numbered as shown in Fig. 8 for a proper implementation in the model.

Concerning the experimental procedure, the contact pressures were measured in quasi-statics for each surface. The tests were performed for a total load $\tilde{P}$ varying between 0 and $150 \mathrm{~N}$ for $S_{1}$ and from 0 to $300 \mathrm{~N}$ for $S_{2}$ and $S_{3}$. Each test was repeated four times in order to have a better significance. Then three total loads were considered for comparisons, which are 50,100 and $150 \mathrm{~N}$ for $S_{1}$ and 200, 250 and $300 \mathrm{~N}$ for $S_{2}$ and $S_{3}$. First the pressure distribution was measured as illustrated in Fig. 9 for surface $S_{2}$ at $250 \mathrm{~N}$. The square pixels on the figure have the size of a sensor cell. The pressure range obtained after calibration is given on the right. The crosses are the positions of the tips and the circles describe the base of the spherical punches. Then the contact forces at the tips of the asperities were calculated by integrating the measured pressure inside each local contact area. The contact forces and pressures were saved for each loading case for comparison with the model.

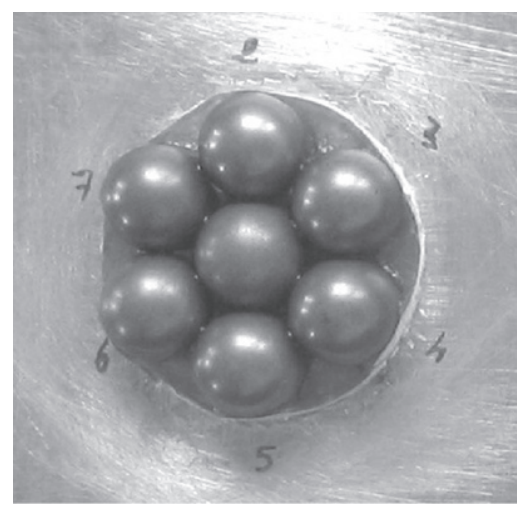

$\mathrm{x}(\mathrm{mm})$

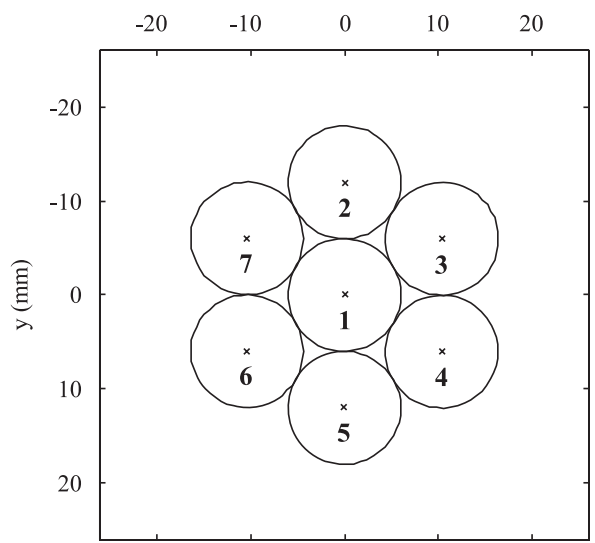

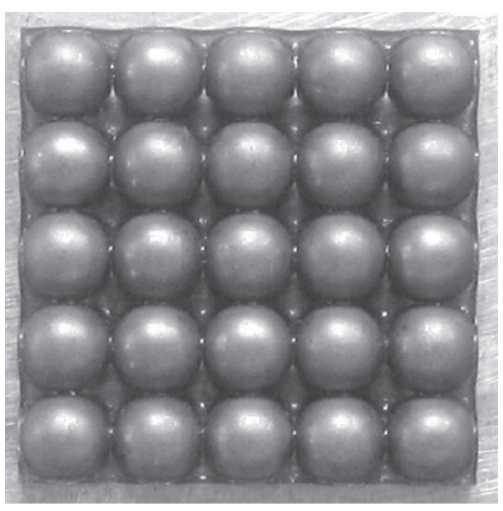

$\mathrm{x}(\mathrm{mm})$

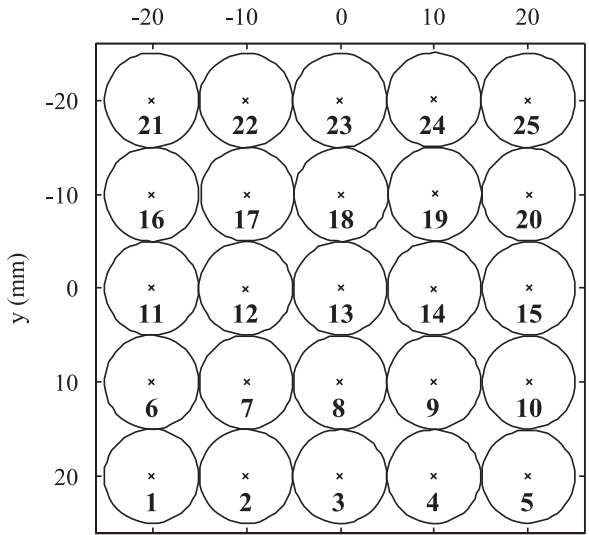

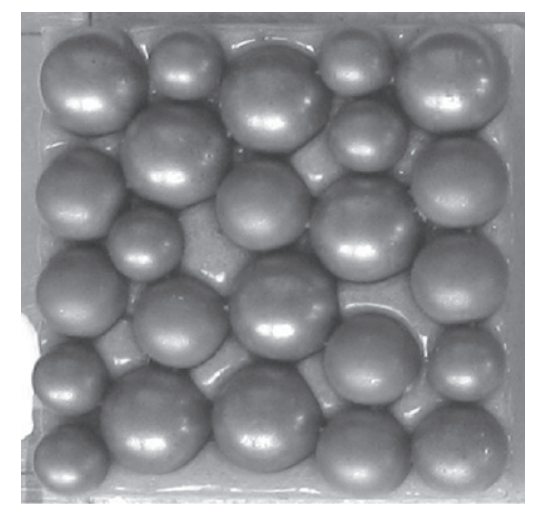

$\mathrm{x}(\mathrm{mm})$

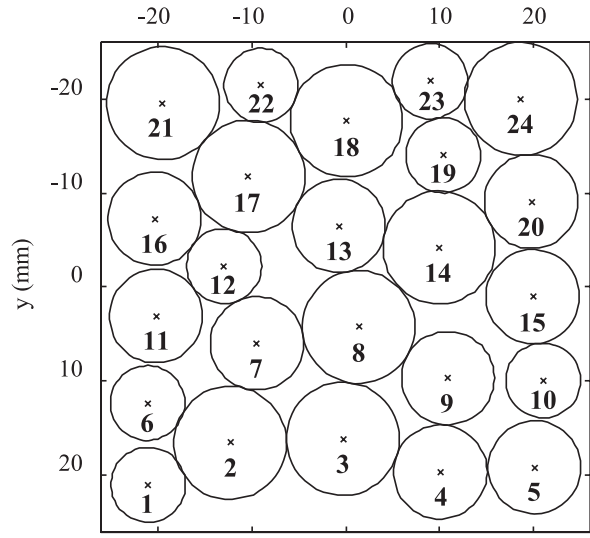

Fig. 8. Pictures of the three indenting surfaces $S_{1}, S_{2}$ and $S_{3}$ (top) and associated numbering (bottom). 


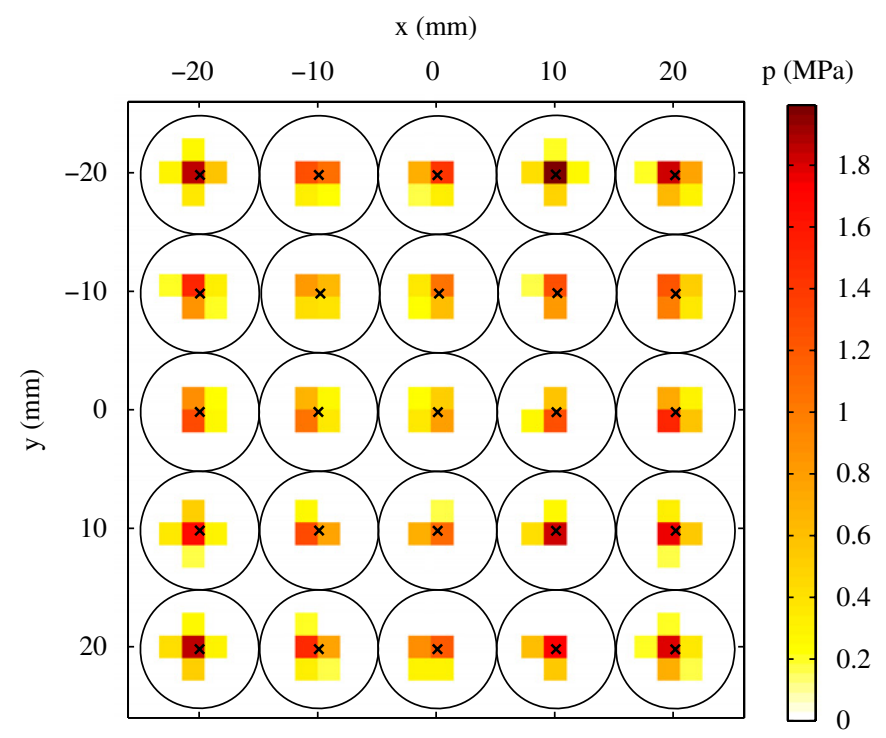

Fig. 9. Contact pressure measurements for the surface $S_{2}$ at $250 \mathrm{~N}$.

\subsection{Modelling implementation}

To model the plain bearings used during the experiments, the macro-scale step of the numerical method was formulated for a known total normal load $P$ with a ball-and-socket joint at its point of application $O$. This enables the surface of the elastic half-space to rotate during loading with angles $\alpha$ and $\beta$, respectively, around the $(\mathrm{Ox})$ and $(\mathrm{Oy})$ axes. Assuming that these angles are small, the local penetration $\delta_{k}$ at point $\left(x_{k}, y_{k}, z_{k}\right)$ can be written as

$\delta_{k}=\delta-z_{k}-\beta x_{k}-\alpha y_{k}-T_{k l} P_{l}$

Then the contact equations can be written as in Eq. (4) where $\delta_{k}$ is replaced by its expression given in Eq. (11). This gives a system of $N$ non-linear equations that contains $N+3$ unknowns which are the $N$ normal contact forces $P_{k}$, the global penetration $\delta$ and the two angles $\alpha$ and $\beta$. Thus three more equations are given by the equilibrium equations in statics:

$\sum_{k=1}^{N} P_{k}=P, \quad \sum_{k=1}^{N} y_{k} P_{k}=0 \quad$ and $\quad \sum_{k=1}^{N} x_{k} P_{k}=0$

The combination of contact equations (4) and equilibrium equations (12) leads to a non-linear system of equations which is solved to give the contact forces. Then the contact pressure can be calculated at a finer scale using the micro-scale calculation step. The fine pressure distribution is next averaged on square elements of the same size as the sensor cells. This enables a rigorous comparison between calculated and measured pressures.

Concerning the input data entered in the model, the total load $P$ was given by the measured one $\tilde{P}$ for each test. The number of asperities $N$ was given by the numbers of spherical indentors for each surface. Then for each punch the load-penetration relation $f_{k}$ was described using Hertz's relation given in Eq. (10). Young's modulus $E$ was equal to the value $6 \mathrm{MPa}$ estimated for the rubber block and Poisson's coefficient $v$ was equal to 0.5 . The geometrical properties of the contacting bodies were also carefully implemented in the model. A three-dimensional mapping of the contacting surfaces was carried out using a high resolution micro-topography measurement system. The position $\left(x_{k}, y_{k}, z_{k}\right)$ and the radius $R_{k}$ of each asperity were locally calculated by approaching the surface data with a sphere using a least mean square procedure. The measured surface of the rubber block was slightly curved which was taken into account in the model by adding it to the height of the asperities. Finally, the coordinates $\left(x_{0}, y_{0}\right)$ of point $O$ could vary during experiments due to the uncertainties in the centering of the plain bearings. These coordinates were calculated for each test from the measured local forces $\tilde{P}_{i}$ assuming that the sum of moments at point $O$ was experimentally null:

$x_{0}=\frac{1}{\tilde{P}} \sum_{k=1}^{N} x_{k} \tilde{P}_{k} \quad$ and $\quad y_{0}=\frac{1}{\tilde{P}} \sum_{k=1}^{N} y_{k} \tilde{P}_{k}$

Then the tips of the asperities were shifted as follows: $\left(x_{k}-x_{0}, y_{k}-y_{0}, z_{k}\right)$. The main consequence is that each test is unique due to centering uncertainties and must be individually compared with the model.

\subsection{Results}

The two-scale iterative method was implemented as described above for each indenting surface and each loading case. Then the predicted values were compared to the measured data both for contact forces and pressure distribution.

At the macro-scale, the numerical contact forces $P_{k}$ were compared to the measured contact forces $\tilde{P}_{k}$. The comparisons for the three loading cases are given in Fig. 10 for surface $S_{1}$ and in Fig. 11 for surfaces $S_{2}$ and $S_{3}$. Fig. 10 for surface $S_{1}$ shows that the measured central force was smaller than the peripheral forces for the three total loads. The same observation was made in Fig. 11(a) for surface $S_{2}$. The smallest force was obtained in the middle of the surface (punch 13) while the highest were found in the corners (punches 1, 5, 21 and 25). This is the proof that an interaction between the punches exists and is not negligible as it was already underlined in the modelling part.

For surface $S_{1}$, the differences between predicted and measured forces were below $10 \%$ for $P=50 \mathrm{~N}$ and below $5 \%$ for $P=$ 100 and $150 \mathrm{~N}$. For surfaces $S_{2}$ and $S_{3}$, the differences were below $10 \%$ for most asperities but could be slightly higher than $10 \%$ for some punches. These correlations are fairly acceptable by keeping in mind both the precision of the measurement system and the simple hypotheses of the model. The differences may be explained by small errors in the centering and in the topography of the contacting surfaces. The topography of the rubber block was actually measured in free conditions which may not exactly correspond to the initial contact conditions. Moreover, a shift in

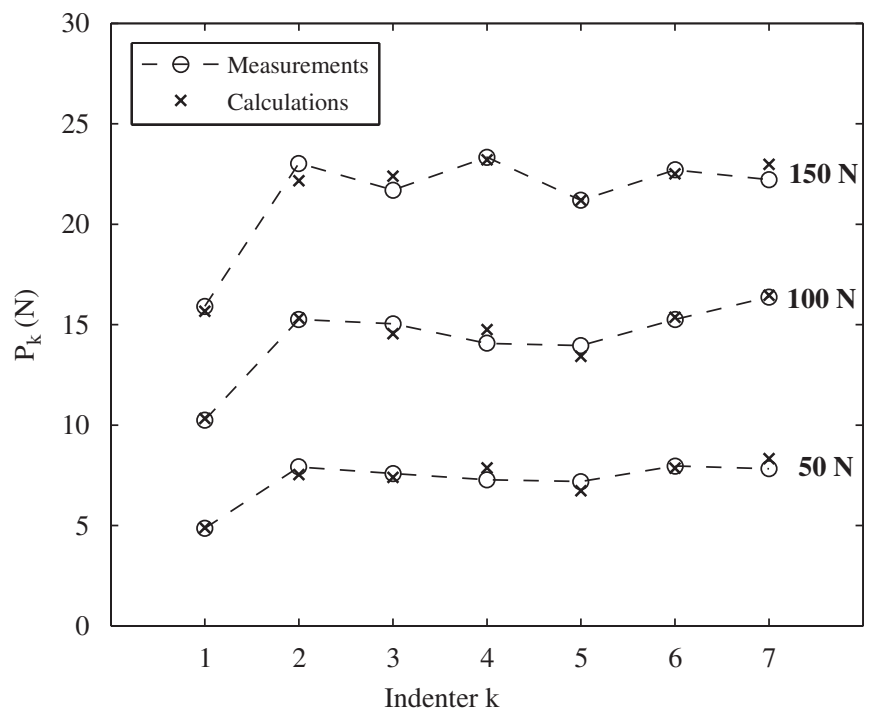

Fig. 10. Comparison of contact forces for the surface $S_{1}$. 
a
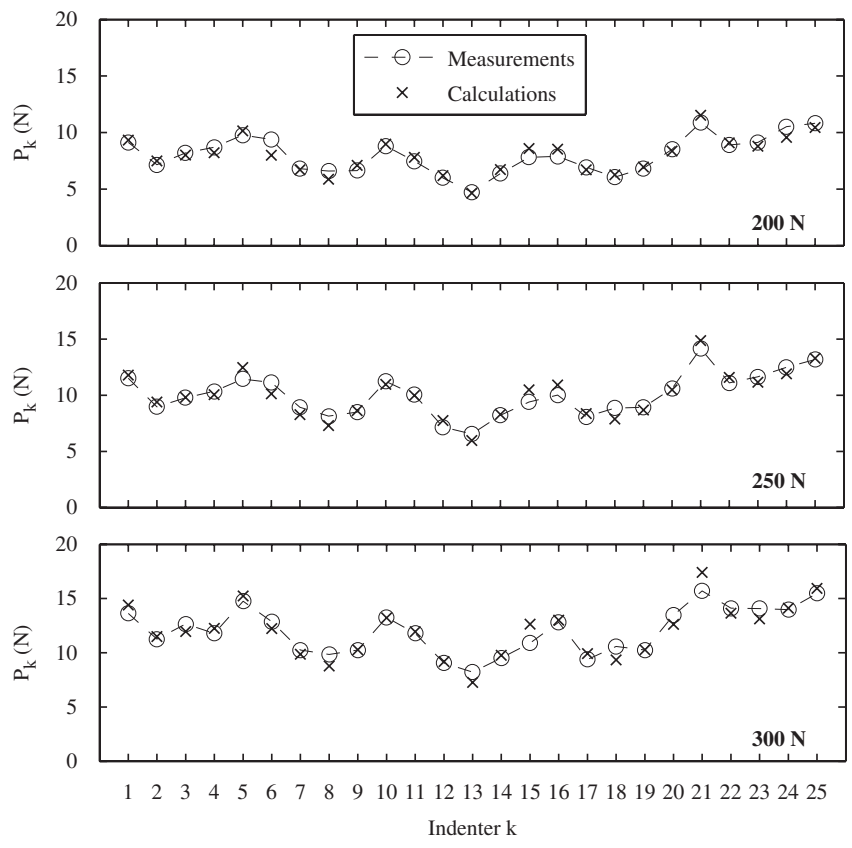

b
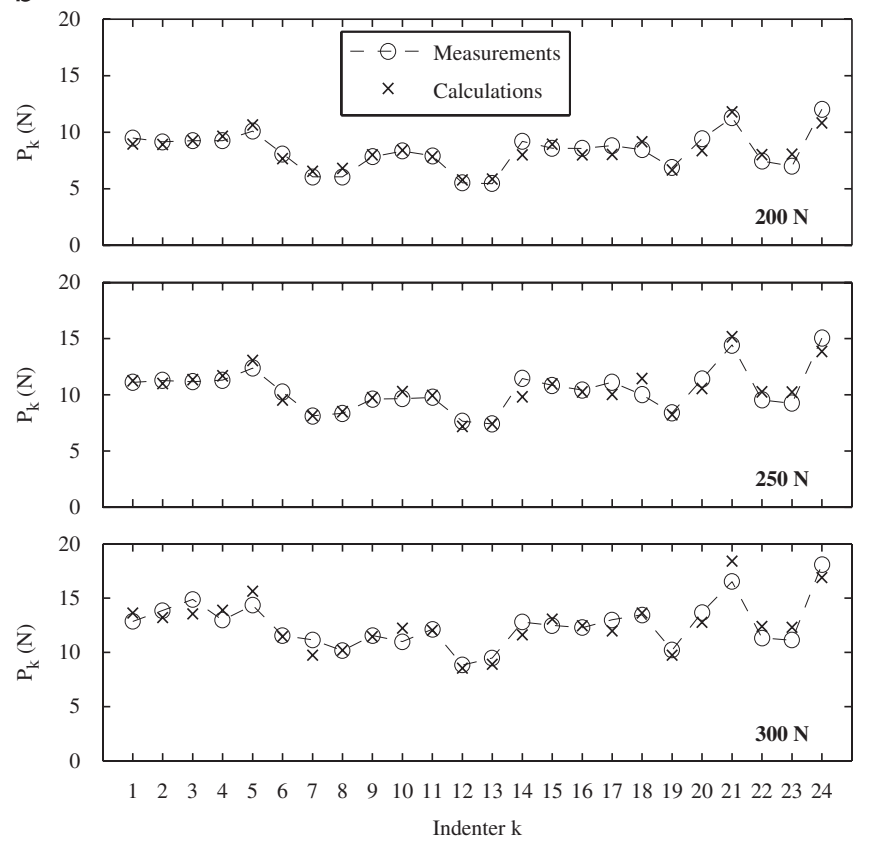

Fig. 11. Comparison of contact forces for the surfaces (a) $S_{2}$ and (b) $S_{3}$.

the relative position of the surfaces can introduce errors in the description of the height of the asperities.

Finally, the macro-scale differences were assessed using the global indicator $\varepsilon_{M}$ defined as follows:

$\varepsilon_{M}=100 \sum_{k=1}^{N}\left|P_{k}-\tilde{P}_{k}\right|^{2} / \sum_{k=1}^{N}\left|\tilde{P}_{k}\right|^{2}$

where $N$ is the number of punches. At the micro-scale, the differences between pressure values were also evaluated using the following indicator $\varepsilon_{m}$ :

$\varepsilon_{m}=100 \sum_{i=1}^{n}\left|p_{i}-\tilde{p}_{i}\right|^{2} / \sum_{i=1}^{n}\left|\tilde{p}_{i}\right|^{2}$
Table 1

Macro- and micro-scale indicators for the three surfaces at the three loading cases.

\begin{tabular}{|c|c|c|c|c|c|c|c|c|c|}
\hline Surface & $S_{1}$ & & & $S_{2}$ & & & $S_{3}$ & & \\
\hline$P(\mathrm{~N})$ & 50 & 100 & 150 & 200 & 250 & 300 & 200 & 250 & 300 \\
\hline $\bar{\varepsilon}_{M}(\%)$ & 0.4 & 0.2 & 0.1 & 0.5 & 0.5 & 0.6 & 1.1 & 0.7 & 0.7 \\
\hline $\bar{\varepsilon}_{m}(\%)$ & 5.9 & 4.8 & 4.9 & 3.9 & 4.2 & 4.0 & 7.4 & 7.4 & 7.0 \\
\hline
\end{tabular}

where $n$ is the number of loaded cells, $p_{i}$ are the calculated pressures and $\tilde{p}_{i}$ are the measured pressures. The indicators were calculated and averaged from the four tests for a given surface at a given total load in order to have significant comparisons. These mean values are noted $\bar{\varepsilon}_{M}$ and $\bar{\varepsilon}_{m}$. They are given in Table 1 . For the three surfaces, the macro-scale indicator was below $1 \%$ for the three total loads, which means that the contact forces are globally in very good agreement. At the micro-scale, $\bar{\varepsilon}_{m}$ was below $10 \%$ for the three configurations and the three surfaces, which means that globally the calculated and the measured pressure distributions were of the same order. Since the precision of the measurements is $\pm 10 \%$, the results show that the proposed method could be relevant to model multi-contact problems at both scales.

\section{Conclusion}

A two-scale iterative method has been developed for solving the problem of multiple contacts at the surface of an elastic halfspace. Numerical results for two and seven spherical indentors show that the method is as accurate as the matrix inversion method [6], even for close proximity contacts. Moreover, the method is very time efficient which will probably make possible the calculation of contact pressure on large surfaces with a great number of indentors.

Concerning the experimental part, the differences between predicted and measured results were below $10 \%$ for different configurations of spherical indentors loaded on a rubber block. These results are fairly acceptable considering the $\pm 10 \%$ precision of the measurement system specified by the manufacturer. The discrepancies observed for some punches were linked to errors or shifting in the description of the surface of the rubber block. The higher average differences for surfaces $S_{2}$ and $S_{3}$ may be explained by the finite dimensions of the rubber block which could be introduced in the model using a finite element model of the block for the calculation of the influence coefficients.

The application of the method on larger surfaces involving punches of random shape is in progress. Further developments of the method should include the consideration of friction between the indentors and the half-space. The viscoelastic behavior of the half-space should also be taken into account so that the method can be applied to practical contact problems, such as tyre-road contact problems. The results obtained in this study show that the two-scale iterative method can be a reliable tool for the calculation of contact forces and stresses in applications where a precision of $10 \%$ is acceptable. For instance, this is the case for the prediction of contact stresses for tyre/road noise modelling, where a difference of $10 \%$ in the contact forces will induce differences below $1 \mathrm{~dB}$ in the noise level. For applications which need higher accuracy, a comparison of the model involving contact areas and pressure measurements with a sensor of finer resolution is required.

\section{References}

[1] Wullens F, Kropp W. A three-dimensional contact model for tyre/road interaction in rolling conditions. Acta Acustica 2004;90:702-11. 
[2] Cesbron J, Anfosso-Lédée F, Duhamel D, Yin HP, Le Houédec D. Prediction of contact stresses for tyre/road noise modelling. In: Proceedings of Euronoise 2006, Tampere, Finland, 30 May-1 June 2006.

[3] Boussinesq J. Application des potentiels à l'étude de l'équilibre et du mouvement des solides élastiques. Paris: Gauthier-Villars; 1885.

[4] Sneddon IN. The relation between load and penetration in the axisymmetric Boussinesq problem for a punch of arbitrary profile. International Journal of Engineering Science 1965;3:47-57.

[5] Greenwood JA, Williamson JBP. Contact of nominally flat surfaces. Proceedings of the Royal Society, London. Series A 1966;295.

[6] Johnson KL. Contact mechanics. Cambridge: Cambridge University Press; 1985.

[7] Kalker JJ, Van Randen Y. A minimum principle for frictionless elastic contact with application to non-Hertzian half-space contact problems. Journal of Engineering Mathematics 1972;6(2):193-206.

[8] Singh KP, Paul B. Numerical solution of non-hertzian elastic contact problems. ASME Journal of Applied Mechanics 1974:96:484-90.

[9] Webster MN, Sayles RS. A numerical model for the elastic frictionless contact of real rough surfaces. ASME Journal of Tribology 1986;108:314-20.

[10] Ju Y, Zhen L. A full numerical solution for the elastic contact of threedimensional real rough surfaces. Wear 1992;157:151-61.
[11] Chang L, Gao Y. A simple numerical method for contact analysis of rough surfaces. ASME Journal of Tribology 1999;121:425-32.

[12] Nowell D, Hills DA. Hertzian contact of ground surfaces. ASME Journal of Tribology 1989;111:175-9.

[13] Ciavarella M, Hills DA, Moobola R. Analysis of plane and rough contact subject to a shearing force. International Journal of Mechanical Sciences 1999;41:107-20.

[14] Chen B, Lang EJ, Chou T-W. Experimental and theoretical studies of fabric compaction behavior in resin transfer molding. Materials Science and Engineering 2001;A317:188-96.

[15] Grenouillat R, Leblanc C. Simulation of mechanical pressure in a rubber-glass contact for wiper systems. SAE Technical Paper Series 2002-01-0798.

[16] Montmayeur N, Choi H-Y, Sah SS, Marca C. Experimental and numerica analyses of seating pressure distribution patterns. SAE Technical Papers Series 2005-01-2703.

[17] Love AEH. Stress produced in a semi-infinite solid by pressure on part of the boundary. Philosophical Transactions of the Royal Society 1929;A228:37-43.

[18] Hertz H. Über die Berührung fester elastischer Körper. Journal Fur Die Reine und Angewandte Mathematik 1882;92:156-71.

[19] Tekscan, Inc. I-Scan ${ }^{\circledR}$ Pressure measurement system-user manual, Version 5.7x. South Boston, 2005. 